\title{
Tecnologias de Fabricação Digital para o Desenvolvimento de Artefatos Responsivos
}

\author{
Digital Manufacturing technologies for Responsive Artifacts Development \\ Waldo Luiz Costa -Neto \\ UFRGS, Brasil \\ waldooo@gmail.com \\ Alexandre Monteiro Barros \\ UFRGS, Brasil \\ alembarros@gmail.com \\ - Vanessa Baldin Gallardo \\ UFRGS, Brasil \\ vavagallardo@gmail.com \\ Underléa Miotto Bruscato \\ UFRGS, Brasil \\ arq.leiab@gmail.com
}

\begin{abstract}
This paper presents the development of a responsive object that addresses issues concerning to digital manufacturing processes as dynamic geometric modelling, where you can check formal and functional aspects of the product; the use of mechanisms for interaction between user and product, through sensors, controllers and their respective programming; and manufacturing using $C A D$ / CAM resources. The aim is to develop a system for responsive objects development through digital manufacturing technologies in order to contribute for concepts implementation in new products design.
\end{abstract}

Keywords: Parametric Design, Responsive Artefact, Digital Manufacturing, Design Product

\section{Introdução}

Artefatos responsivos são caracterizados pela alteração de suas propriedades de maneira programada quando determinados fatores são modificados. Esta adaptação pode acontecer de diferentes modos, como alterações de forma, cor, textura ou som. Os fatores que desencadeiam esta resposta podem ser associados a mudanças de posição do objeto, de tempo, de proximidade a um alvo, entre outros (REBAUDENGO, 2012). Em Soft Architecture Machines (1975), Nicolas Negroponte define Arquitetura Responsiva como sinônimo de reativa, ou adaptável e na qual "o ambiente tem um papel ativo, dando início a mudanças de maior ou menor grau, como resultado e em função de computações complexas ou simples." (tradução livre do original em inglês, NEGROPONTE, 1975). (NEGROPONTE, 1975)

Segundo Tristan d'Estrée Sterk ${ }^{1}$, a "arquitetura responsiva funcional ideal é a que providencia abrigo contra condições variáveis, bem como calcula como essas variáveis afetam o tipo de abrigo necessário."(STERK, 2003) É uma arquitetura que reflete a tecnologia e cultura do nosso tempo, em que o uso do computador está sendo cada vez mais usado não apenas como um instrumento de representação de projeto,

1 (Sterk, Tristan d'Estrée - Using Actuated Tensegrity Structures to Produce a Responsive Architecture. The School of The Art Institute of Chicago, USA.) mas com maior importância nas fases de desenvolvimento através do uso de softwares paramétricos e na criação de modelos para fabricação digital.

O desenvolvimento e a difusão de novas tecnologias para a programação e o controle de dispositivos eletrônicos, em especial as plataformas open-source, com softwares e hardware acessíveis a um maior número de projetistas vem mudando este cenário. Novos produtos vêm sendo desenvolvidos de forma independente justamente pela maior acessibilidade aos equipamentos e ferramentas deste tipo de produto. Um exemplo são os produtos "viciados" (addicted products), uma linha de pesquisa que propõe a conexão de eletrodomésticos em rede e a comunicação destes artefatos com os usuários baseada na quantidade de vezes que o aparelho é utilizado. Esta comunicação é feita mediante a resposta através de movimentos que pretendem indicar satisfação após utilizados ou, ao contrário, solicitar durante o seu uso. O objetivo é que os equipamentos, através da interação, estimulem o uso e uma relação emocional com o proprietário (Figura 1), como observado por Rebaudengo (2012):

"Nós tentamos projetar um sistema no qual as pessoas não são convidadas a mudar e os produtos não dominem ou digam o que devemos fazer, mas sim que eles apenas possam mostrar um novo caminho e façam nós percebermos o fato de que os produtos, mesmo o mais elementar deles, têm um custo e são 
relevantes em nossas vidas" (REBAUDENGO, 2012).

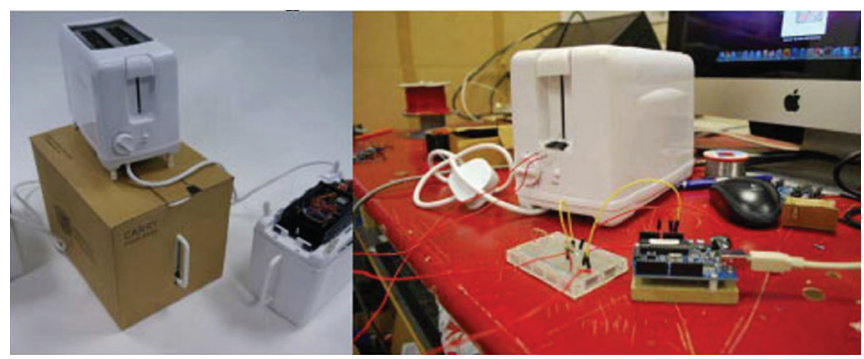

Figura 1: Addicted Product. Rebaudengo (2012)

Grandes empresas também vêm investindo nesta categoria de produto, que integra interação e respostas direcionadas ao usuário. De acordo com Alonso et al. (2011), ao longo dos últimos anos o design de produto começou a incorporar produtos interativos, capazes de responder e se adaptar ao comportamento do usuário. Conforme Desmet e Hekkert (2007), a interação entre usuário e produto pode acontecer em três níveis. O primeiro nível é a interação instrumental, baseada no manuseio dos dispositivos. $\mathrm{O}$ segundo é a interação não instrumental, definida pela relação entre usuário e produto sem operar uma função específica. $O$ terceiro nível consiste na interação não física, sem contato com o produto. Um exemplo de produto interativo é a luminária Wake-up ${ }^{\circledR}$ da empresa Philips ${ }^{\circledR}$ (Figura 2). Esta luminária pode ser programada para fornecer uma luminosidade e uma sonoridade gradual com o objetivo de proporcionar uma maneira mais amigável e natural no despertar.

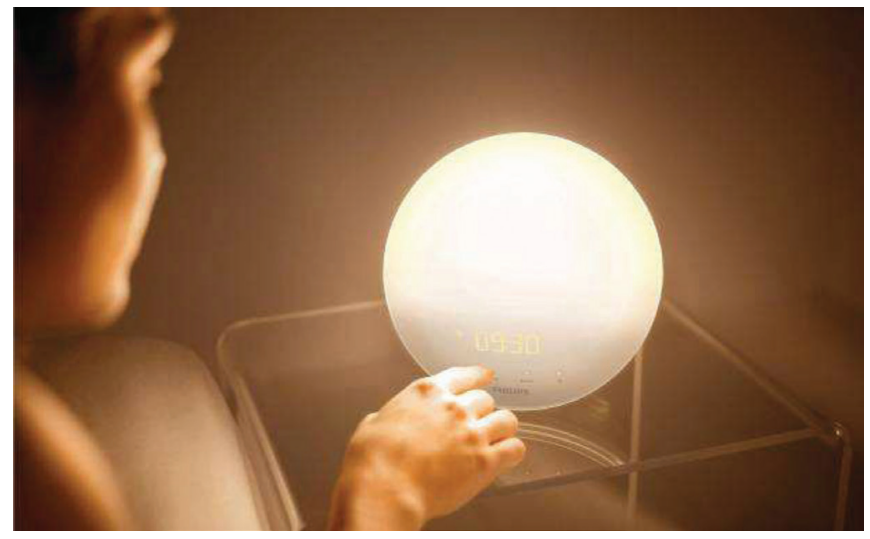

Figura 2: Wake-up $®$ Light. Philips ${ }^{\circledR}(2013)$

Ao mesmo tempo em que as tecnologias eletrônicas que permitem desenvolver produtos responsivos estão mais acessíveis para um maior número de projetistas, os processos de produção associados às tecnologias computacionais também vêm permitindo uma maior aproximação entre o projeto e a manufatura dos artefatos, viabilizando a experimentação em diversas áreas do design de produto e arquitetura (BRUSCATO, 2011). As técnicas de modelagem computacional integram diferentes tipos de modelos para representar, manipular, analisar e materializar os artefatos. Os modelos conceituais, paramétricos, generativos, de desempenho e os modelos construtivos fornecem um amplo campo de investigação e aplicação para o projeto de produtos e sistemas. A Figura 3 demonstra uma sequência de aplicação dos modelos em função das etapas do projeto elaborada por Kilian (2006). Os artefatos podem se adaptar a diferentes entradas de dados, analisados em relação ao seu desempenho, gerar variações geométricas através de algoritmos específicos, simular comportamentos físicos, bem como organizar e orientar a fabricação e o funcionamento de seus componentes constituintes.

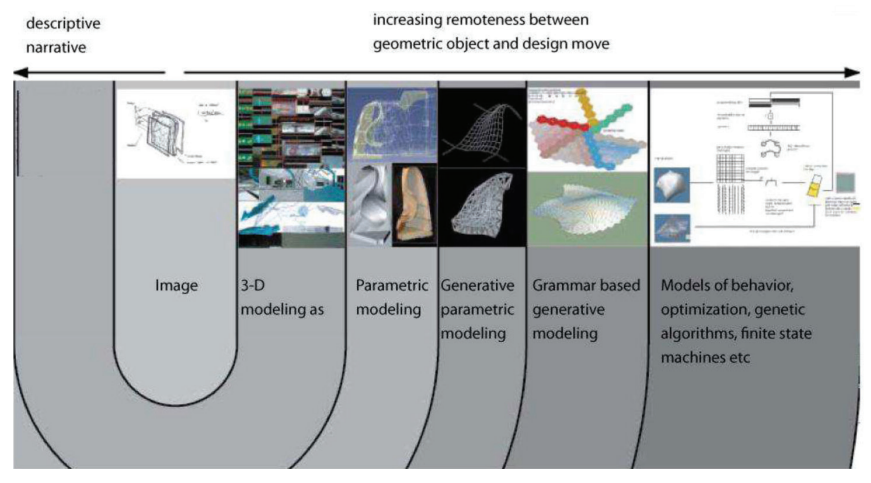

Figura 3: Aplicação de modelos no desenvolvimento de projeto. Kilian (2006)

Os equipamentos que fazem parte das tecnologias de fabricação digital são associados aos sistemas de controle numérico computadorizado (CNC). Os principais tipos são as cortadoraslaser, as routerseasimpressorastridimensionais, que interpretam modelos digitais fazendo a transposição do virtual para o material de forma precisa e direta. Embora os sistemas construtivos dos objetos sejam diferentes para cada tecnologia empregada, estes sistemas possibilitam uma grande variação formal, facilitando a prototipagem e, conseqüentemente, o desenvolvimento de novos produtos a custos acessíveis, pois já é um maquinário presente tanto em indústrias quanto em instituições de ensino e pesquisa. Considerando o contexto apresentado e estimando a possibilidade de contribuir para o desenvolvimento tecnológico na área abordada, foi estruturado e desenvolvido um experimento visando sistematizar as ferramentas e as tecnologias acima mencionadas para o projeto e a produção de artefatos responsivos, de modo a possibilitar o seu uso no design de novos sistemas e produtos através de um caminho sugerido.

\section{Desenvolvimento}

O processo iniciou com a definição da ideia de produto a ser desenvolvido e a discussão acompanhada de croquis, diagramas e listagem das características das funções desejadas 
(Figura 4 e 5). Os diagramas estão associados ao paradigma da orientação ao objeto (BARROS, SILVA e TEIXEIRA; 2011), possibilitando descrever o contexto, as interfaces e os componentes do artefato.

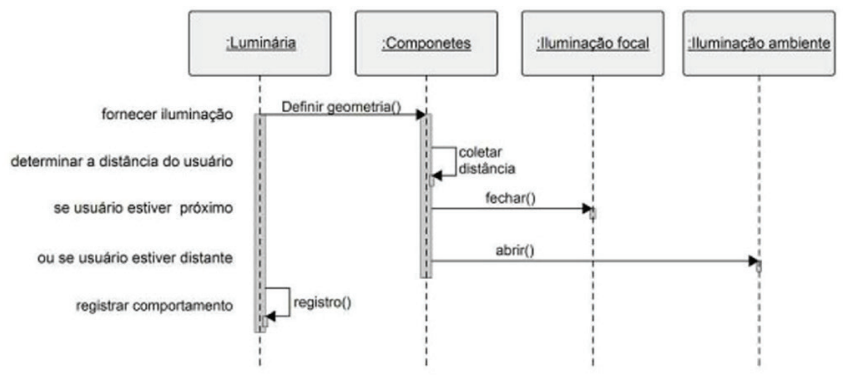

Figura 4: Diagrama de Sequência elaborado.

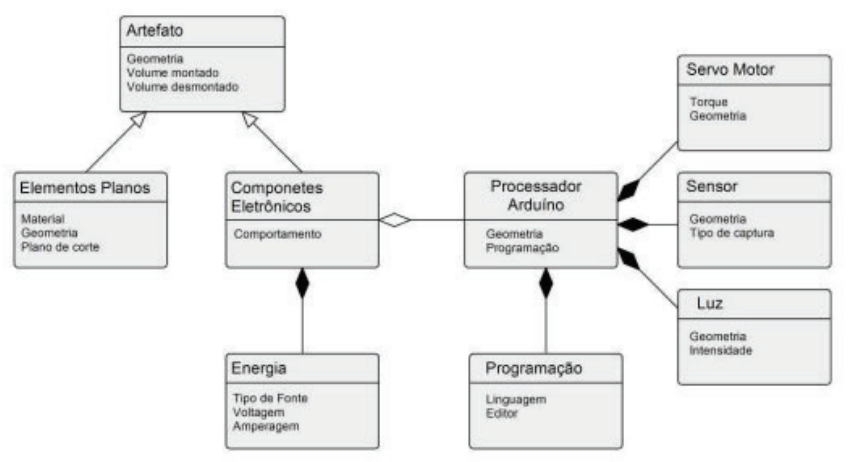

Figura 5: Diagrama de Classes para o Artefato.

A forma do artefato consiste na união de peças triangulares, ou seja, a geometria do objeto foi determinada a partir de uma forma elementar: o triângulo. O triângulo permite alterações plásticas a partir da variação paramétrica, além de ser a forma elementar que mais possibilita o aproveitamento de material. Após a concepção da idéia inicial, a modelagem geométrica do artefato foi realizada no software paramétrico Grasshoper $\circledast$, plugin do programa de modelagem tridimensional Rhinoceros $®$, para o estudo de configurações espaciais e dos movimentos possibilitados ou restringidos pelas formas utilizadas.

Esta modelagem envolveu a inclusão de variáveis relativas à simulação da transformação projetada para a geometria dos sistemas. Na sequência foi feita a modelagem paramétrica tridimensional do artefato, associada aos parâmetros de geometria e às possíveis variações dimensionais provocadas pela interatividade entre o próprio objeto responsivo e o usuário. Um dos condicionantes de projeto introduzidos durante a modelagem geométrica foi que o artefato fosse formado por peças geométricas planas, unidas umas às outras por suas arestas (Figura 6). Por serem planas, ou seja, bidimensionais, poderiam ser fabricadas a partir de chapas cortadas em CNC ou cortadora laser, em acordo com este tipo de sistema construtivo. Com a modelagem paramétrica foi possível simular propriedades e fenômenos físicos pelo Kangaroo $\AA$, como por exemplo, elasticidade,

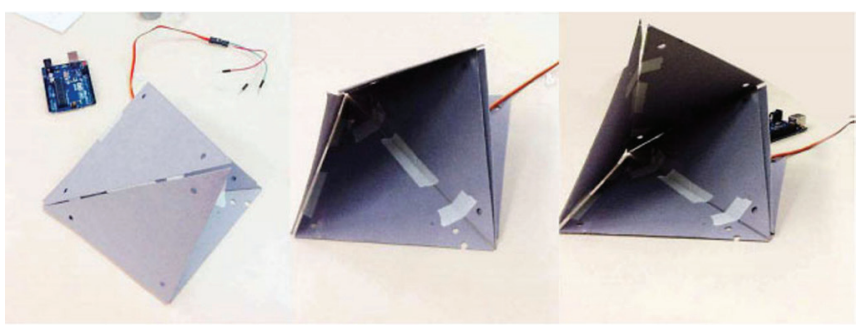

Figura 6: Figura formada por triângulos iguais.

massa, movimento e gravidade.

Após experimentação de diferentes dimensões e proporções chegou-se ao modelo escolhido, formado por triângulos isósceles de base tamanho $20 \mathrm{~cm}$ e lados $15 \mathrm{~cm}$. A junção de triângulos isósceles permitiu a movimentação do objeto e dobras nas arestas, o que possibilita a transformação do volume em superfície e vice-versa, facilitando posteriormente o transporte do produto.

A interação usuário-luminária foi testada através da manipulação de parâmetros introduzidos também no ambiente do Grasshopper ${ }^{\circledR}$ e Kangaroo ${ }^{\circledR}$ (Figura 7) a fim de verificar se a amplitude do movimento de transformação

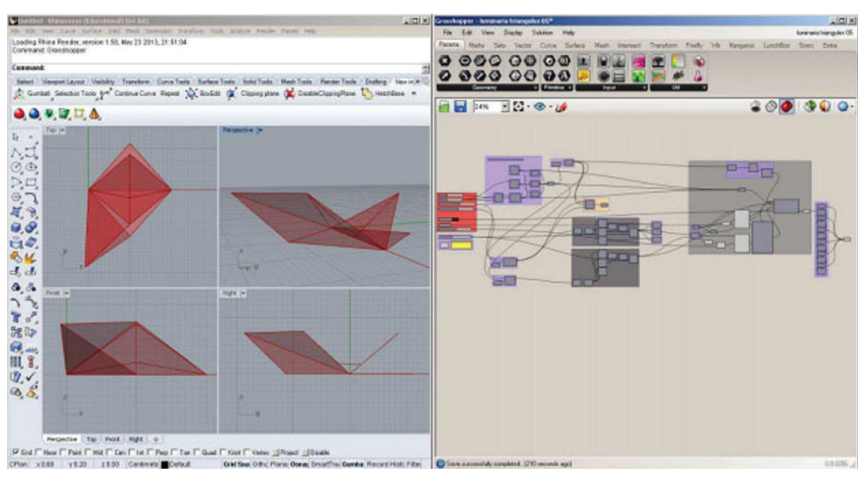

Figura 7: Objeto virtual parametrizado - Grasshoper.

estava criando o efeito desejado.

Verificando os padrões geométricos mais satisfatórios com base nos diferentes critérios mencionados, um primeiro protótipo físico de papelão de um milímetro foi materializado na escala 1:1 (Figura 8). O modelo físico serviu para testar a interação da luminária com o usuário através da instalação de um sensor de distância através de ultrassom direcionado ao usuário e um servo-motor para controlar a transformação de forma. O processamento do 
sinal captado pelo sensor e a respectiva resposta enviada ao servo-motor é feito através do microcontrolador Arduino ${ }^{\circledR}{ }^{2}$, uma plataforma de prototipagem open-source de baixo custo e de fácil programação.

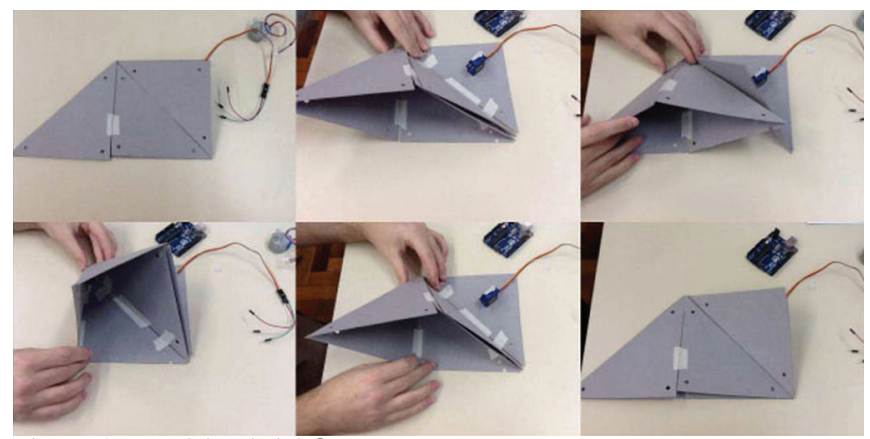

Figura 8: Protótipo inicial 1:1.

O potencial de interação depende da quantidade de sensores, atuadores e do algoritmo criado para controlar o comportamento do objeto. Diferente de um sistema predisposto a iniciar uma ação que dependa diretamente de um sinal recebido por um sensor, um sistema responsivo deve ser capaz de perceber mais informações sobre o contexto em que está inserido, precisa "conhecer" o usuário. (NEGROPONTE, 1975). Um exemplo dado por Negroponte é o de um sistema de controle de temperatura que ao invés de manter uma determinada temperatura e umidade préestabelecidas como confortável para o usuário médio, seria capaz de alterar por ação própria estes fatores a qualquer momento baseado no tipo de atividade e perfil dos usuários presentes no ambiente. (NEGROPONTE, 1975). Para simplificar o experimento, foi mantido apenas um sensor de distância e um servo-motor. Devido a essa limitação no número de sensores e atuadores, segundo a definição de Negroponte (NEGROPONTE, 1975) o objeto criado não seria propriamente Responsivo. Apesar do uso de apenas um sensor e um atuador no objeto proposto limitar o número de estímulos e respostas possíveis, esta foi intencional, pois o objetivo principal está na documentação do processo.

A programação foi feita para que a luminária assumisse duas formas diferentes: (I) aberta iluminando o ambiente quando o usuário mais distante; (II) com a abertura mais fechada, assumindo uma forma mais horizontal para direcionar a luz e proteger o usuário de ofuscamento quando próximo ( $80 \mathrm{~cm}$ ou menos do sensor). A observação do protótipo permitiu a evolução da união flexível entre os elementos, que foi feita para o modelo final através de uma costura próxima às arestas de encontro entre estes e da posição do furo onde passa o cabo puxado pelo servo-motor (e provoca a transformação) que foi ajustada para o tamanho do servo utilizado. A fase seguinte foi atualizar o modelo paramétrico a partir das

2 Desenvolvido por Massimo Banzi, David Cuartielles, Tom Igoe, Gianluca Martino, Daniela Antonietti e David A. Mellis. Disponível em: http://www.arduino.cc/ conclusões obtidas através do modelo físico e a preparação do arquivo para corte do produto final. Diferentemente do protótipo que havia sido cortado em papelão de $1 \mathrm{~mm}$ de espessura, para o produto final utilizamos papelão ondulado de face dupla (espessura $2 \mathrm{~mm}$ ) cortado e perfurado na máquina laser. Todas as peças foram fabricadas da mesma forma para que o produto final pudesse ser montado facilmente e em número maior do que o necessário, possibilitando assim novas combinações criadas pelo próprio usuário. Após a finalização do experimento, um levantamento das questões foi realizado
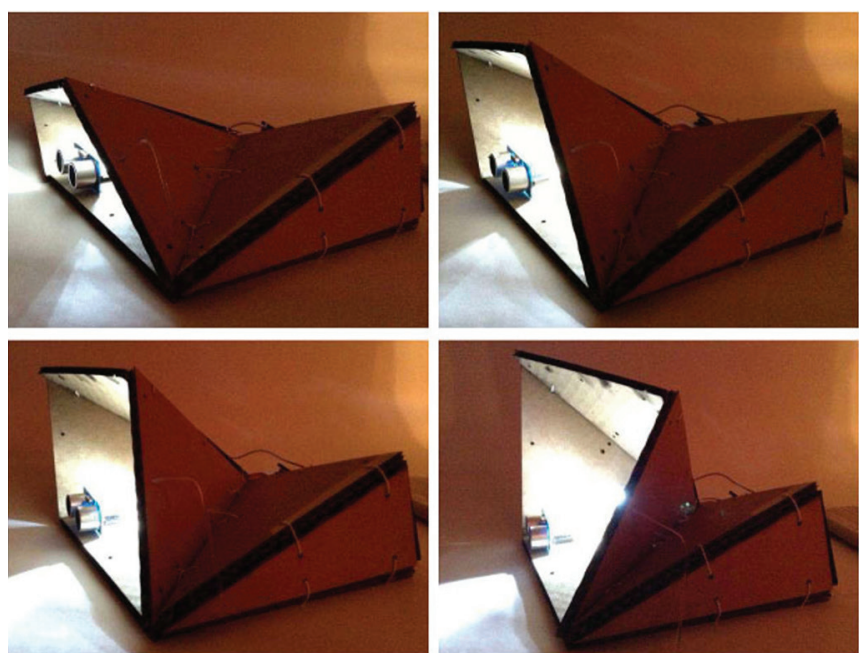

Figura 9: Protótipo Final 1:1

para apresentar o sistema proposto.

\section{Resultados e Discussão}

O experimento desenvolvido resultou em uma sistematização das técnicas e das ferramentas aplicadas no projeto e na prototipagem de um produto responsivo. Deste modo, considerando as etapas básicas de projeto de produto, a

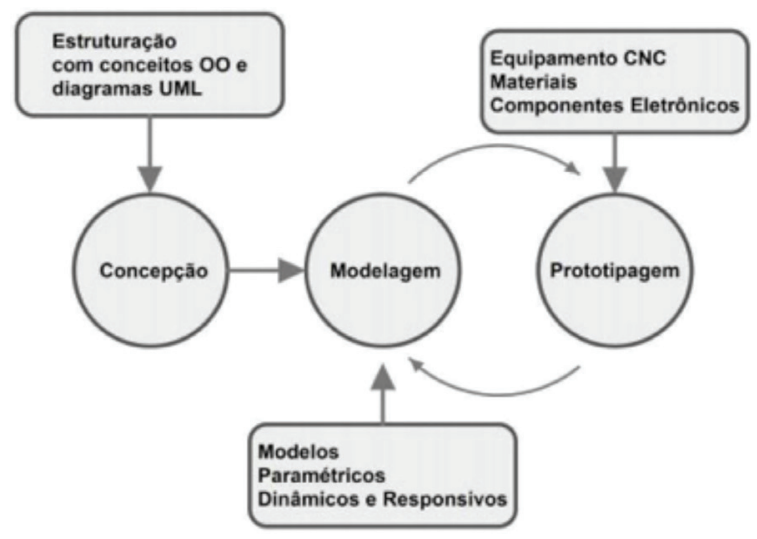

Figura 10: Sistema para o desenvolvimento de Artefatos Responsivos. 
figura 10 apresenta a sistematização empregada.

Deste modo, conforme apresentado durante o desenvolvimento do experimento, as técnicas e ferramentas empregadas possibilitaram trabalhar no problema abordado de maneira sistemática e com resultados satisfatórios para a materialização do produto. Em relação a solução de projeto adotada utilizando o sistema proposto, foi possível constatar determinados fatores que podem ser aperfeiçoados para a produção definitiva deste produto, caso for necessário:

- $\quad$ O servo-motor não obteve o desempenho adequado ao funcionamento dos movimentos responsivos no segundo modelo, portanto deverá ser substituído por um servo-motor com maior torque, se mantido o material mais espesso.

- $\quad$ A utilização de um material mais espesso teve a intenção de verificar questões formais. Porém, o resultado obtido mostra que a espessura prejudicou o funcionamento pleno entre as articulações, devendo atentar para este fator em relação ao movimento do artefato.

- $\quad$ Novos protótipos devem ser gerados e avaliados apara viabilizar a produção em série deste produto. Entretanto, os avanços necessários são questões relativas a seleção de materiais, conexões e aprimoramento formal do artefato.

\section{Conclusão}

Os processos de fabricação digital facilitam o desenvolvimento de objetos desde a confecção dos protótipos de estudo até a produção em série do produto final. No experimento realizado os processos de CAD (Computer Aided Design) e CAM (Computer Aided Manufacturing) foram ferramentas fundamentais para o desenvolvimento do objeto produzido, neste caso especificamente, uma luminária responsiva. No ambiente $\mathrm{CAD}$, com o auxílio do software paramétrico Grasshoper ${ }^{\circledR}$ e seu plug-in Kangaroo ${ }^{\circledR}$ foi possível desenvolver com precisão o projeto das peças geradoras do objeto para o ambiente CAM, onde foram produzidas através de corte a laser, por tratar-se de uma geometria planificável. Tanto a parametrização, que possibilita a geração de modelos com formas e dimensões variadas a partir do modelo parametrizado inicialmente, quanto a simulação de movimentos no ambiente virtual, que permitiram a definição precisa dos parâmetros de corte, foram relevantes para a fabricação do produto final desse experimento e devem ser considerados no desenvolvimento de produtos responsivos.

\section{Agradecimentos}

Gostaríamos de agradecer ao Professor Dr. Benamy Turkienicz da Universidade Federal do Rio Grande do Sul, UFRGS.

\section{Referências}

BARROS, A; SILVA, R; TEIXEIRA, F. Processo de Projeto de Produto a partir do Paradigma da Orientação a Objetos. $10^{\circ}$ P\&D Design - Congresso Brasileiro de Pesquisa e Desenvolvimento em Design, 2012.

BRUSCATO, U. M. Práticas digitais na arquitetura: Seis experiências internacionais. Arquitetura Revista. Unisinos, São Leopoldo, 2011.

CUBIFY. Cube: 3D Printer [Internet], 2013. 3D Systems ${ }^{\circledR}$. Disponível em:<http://cubify.com/cube/ Acesso em 25 de junho de 2013.

DESMET, P; HEKKERT, P. Framework of product experience. International Journal of Design, 1(1), 57-66, 2007.

KILIAN, A. Design Exploration through Bidirectional Modelling of Constraints. These (PhD. Design and Computation) Massachusetts Institute of Technology, Dept. of Architecture, Massachusetts, 2006.

REBAUDENGO, S. A scenario of future interactions based on addictions of products to be used. 2012. Design \& Emotion. AWARDS, 8TH INTERNATIONAL CONFERENCE ON DESIGN \& EMOTION, LONDON 11-14 SEPT. 2012.

KOLAREVIC, B. Architecture in the digital age: Design and Manufacturing. Taylor \& Francis, 2005.

IWAMOTO, L. Digital fabrications: Architectural and material techniques. Princeton Architecture Press, 2009.

NEGROPONTE, N. Soft Architecture Machines. Cambridge, Mass: The MIT Press, 1975.

STERK, T. D. Using Actuated Tensegrity Structures to Produce a Responsive Architecture. ACADIA22: Connecting Crossroads of Digital Discourse, p. 84-93, 2003. 